\title{
Feasibility Condition for the Uplink of a CDMA Multiservice Mobile Communication System with Repeaters
}

\author{
Luis Alonso, Member, IEEE, Mario Garcia, and Fernando Casadevall, Member, IEEE
}

\begin{abstract}
This paper derives a general analytical expression of the feasibility condition for the uplink of a CDMA mobile communications system in a general scenario where the users in the system belong to an arbitrary number of service classes and the coverage deployment includes the presence of repeaters. An explicit expression of the admission region is derived and the dependencies on the parameter values are shown. An example is presented in order to illustrate the application of the proposed methodology in a real case.
\end{abstract}

Index Terms-CDMA, mobile communications, capacity, multi-service.

\section{INTRODUCTION}

$\mathbf{M}$ OBILE communications systems often work under high heterogeneous conditions, both in time and space domains. This fact makes the proper design of the network infrastructures very important, specially the radio access part. Optimizing this design is a technical and economical challenge. On the other hand, active repeaters are of special interest when considering the deployment of the coverage of a mobile communications system in a certain set of situations that include, but are not limited to: Inside big buildings, tunnels, or any place where the propagation losses are high, open areas where there are time limited very high demand peaks (stadiums, expositions, etc), remote sites, normally in rural areas, where the service demand is widely spread in space domain, suburban areas, near big urban cities, and roads and railroads.

The presence of repeaters modifies the interference and thermal noise patterns. Note that, using the load factor definition in [1], the presence of a single repeater in a cell increases the load factor up to 0.5, even in the absence of active users. Although much previous research efforts have been focused on the analysis of CDMA-based systems ([2]-[4] only to cite a few), not many studies in the literature analyse the effect of repeaters on the capacity and feasibility conditions for a CDMA mobile communications system [5]-[7]. Moreover, these studies consider simplified and homogeneous scenarios, with the presence of users that belong to a single service class. It is clear that, in the near future, mobile communication

Manuscript received June 23, 2004. The associate editor coordinating the review of this letter and approving it for publication was Prof. Gianluca Mazzini. This work was supported by Telefonica Moviles.

Luis Alonso, Mario Garcia, and Fernando Casadevall are with the Department of Signal Theory and Communications of the UPC (e-mail: \{luisg, mariogarcia, ferranc\}@tsc.upc.edu).

Digital Object Identifier 10.1109/LCOMM.2005.05024. systems will support multimedia services with diverse traffic patterns, distributions and QoS requirements. Having all these ideas in mind, this paper derives the analytic expression of the feasibility condition for the uplink of a CDMA mobile communications system with repeaters in a multi-service environment with a general heterogeneous layout.

\section{System Design}

Consider a general mobile communications system based on a CDMA access scheme with an arbitrary number of Base Stations. One of them, the one under study, has $N_{r}$ repeaters connected to it. These repeaters extend the Base Station coverage. We assume that the users in the system belong to one of $M$ possible different services, each one with its own set of QoS parameters. In particular, each service $k(1 \leq k \leq M)$ has a bit rate $\left(R_{b, k}\right)$, activity factor $\left(\nu_{k}\right)$ and required energy per bit versus total interference plus noise ratio $\left(E_{b} / I_{t}\right)_{r e q, k}$. We denote by $N_{b, k}$ the number of users belonging to service $k$ connected directly to the Base Station, and by $N_{m, i, k}$ the number of users belonging to service $k$ connected to each repeater $i$. We assume that each user is connected only through one system element, that is, the Base Station or one of the repeaters.

Let $S_{k}$ denote the received power at the Base Station from any user belonging to service $k$. If a user is connected through a repeater, this value may not be the same as the received power at the repeater. The difference will depend on the repeater settings. However, the quality of the transmission depends only on $S_{k}$ as the power control scheme only uses this target value.

Then, the average quality of the transmissions of the users of service $k,\left(E_{b} / I_{t}\right)_{k}$ can be evaluated by means of the following expression:

$$
E\left[E_{b} / I_{t}\right]_{k}=\frac{S_{k} / R_{b, k}}{\gamma_{b}+\gamma_{r}+\gamma_{u}} \quad k \in\{1, \ldots, M\}
$$

where $E[\cdot]$ stands for the expected value, $\gamma_{b}$ is the spectral density of the thermal noise generated by the Base Station receiver, $\gamma_{r}$ is the spectral density of the thermal noise generated by the receivers of all the repeaters, and $\gamma_{u}$ is the level of interference produced by all the users except the one considered in the evaluation. $\gamma_{b}$ can be calculated from the following expression:

$$
\gamma_{b}=N_{t h} F_{b}
$$


where $N_{t h}$ is the reference thermal noise density $\left(\mathrm{KT}_{0}=-174\right.$ $\mathrm{dBm} / \mathrm{Hz}$ ) and $F_{b}$ is the Base Station Noise Figure. On the other hand, $\gamma_{r}$ can be evaluated as:

$$
\gamma_{r}=\sum_{i=1}^{N_{r}} G_{R X, b} G_{T X, r i, b} G_{r i} L_{b, r i} N_{t h} F_{r i}
$$

where $G_{R X, b}$ denotes the gain of the Base Station receiver for the link with the repeater, $G_{T X, r i, b}$ is the gain of the transmitter of the repeater in the link with the Base Station, $G_{r i}$ is the gain of the repeater $i, L_{b, r i}$ is the propagation loss in the link between the Base Station and the repeater $i$ (considered stationary) and $F_{r i}$ is the Noise Figure of repeater $i$. This expression sets the explicit dependency of the repeaters' configuration on the quality, as the contribution of $\gamma_{r}$ could be even higher than $\gamma_{b}$, even in the absence of active users. Finally, the value of the multiuser interference $\gamma_{u}$ is:

$$
\begin{aligned}
\gamma_{u} & =\frac{(1+\beta)}{W}\left[\nu_{k} S_{k}\left(N_{b, k}+\sum_{i=1}^{N_{r}} N_{m, i, k}-1\right)\right. \\
& \left.+\sum_{n=1}^{M} \nu_{n} S_{n}\left(N_{b, n}+\sum_{i=1}^{N_{r}} N_{m, i, n}\right)\right]
\end{aligned}
$$

where $\beta$ is the intercell interference ratio, supposed constant, and $W$ is the total available bandwidth. Notice that the interference contribution of each user depends on its $S_{k}$ and, assuming that repeaters are properly designed and they not produce distortion due to saturation or other undesirable effects, this interference is independent on whether the user is connected through a repeater or not. Then, the relevance of the thermal noise increase produced by repeaters is stressed as constitutes the main impact on the system.

1) Received Power: The power control scheme will select the appropriate value for each $S_{k}$ in order to achieve the required $\left(\mathrm{E}_{b} / \mathrm{I}_{t}\right)_{r e q, k}$ for each service $k$. These values are obtained from the $M$ equations linear system which is generated applying the expression (1) for each one of the services $k \in\{1, \ldots, \mathbf{M}\}$. In order to simplify the notation, let us make the following definitions:

$$
\begin{gathered}
\alpha_{k} \equiv \nu_{k}+\left(\frac{W}{R_{b, k}}\right) \frac{1}{(1+\beta)}\left(\frac{E_{b}}{I_{t}}\right)_{r e q, k}^{-1} \\
\gamma^{\prime} \equiv \frac{\gamma_{b}+\gamma_{r}}{1+\beta} W \\
\sigma_{k} \equiv \nu_{k}\left(N_{b, k}+\sum_{i=1}^{N_{r}} N_{m, i, k}\right)
\end{gathered}
$$

Note that $\sigma_{k}$ represents the total effective number of users belonging to service $k$, connected to both the Base Station or any of the repeaters. With these definitions, the system of equations can be expressed in matrix notation as follows:

$$
(\Omega-A) \cdot S=-\Upsilon
$$

where:

$$
\Omega=\left[\begin{array}{llll}
\sigma_{1} & \sigma_{2} & \cdots & \sigma_{M}
\end{array}\right]^{T} \cdot\left[\begin{array}{llll}
1 & 1 & \cdots & 1
\end{array}\right]
$$

$$
\begin{gathered}
A=\left[\begin{array}{cccc}
\alpha_{1} & 0 & \cdots & 0 \\
0 & \alpha_{2} & 0 & 0 \\
0 & 0 & \cdots & \cdots \\
0 & 0 & 0 & \alpha_{M}
\end{array}\right] \\
\Upsilon=\gamma^{\prime} \cdot\left[\begin{array}{llll}
1 & 1 & \cdots & 1
\end{array}\right]^{T} \\
S=\left[\begin{array}{llll}
S_{1} & S_{2} & \cdots & S_{M}
\end{array}\right]^{T}
\end{gathered}
$$

This linear system of equations allows to obtain the optimum received power for each service $\left(S_{k}\right)$, which assures the QoS, when all the rest of parameters are known, including the number of users belonging to each class $\left(\sigma_{k}\right)$.

It is possible to generalize the solution of this system for any number of services $(\mathrm{M})$. Indeed, developing the determinant of the matrix $(\Omega-\mathrm{A})$ and working with the obtained expressions, we can derive that the needed receiver power for all users belonging to service class $\mathrm{k}$ can be expressed as:

$$
S_{k}=\gamma^{\prime} \frac{\prod_{j \neq k}^{M} \alpha_{j}}{\prod_{j=1}^{M} \alpha_{j}-\sum_{j=1}^{M} \sigma_{j} \prod_{m=1}^{M} \alpha_{m \neq j}}=\frac{\gamma^{\prime}}{\alpha_{k}\left(1-\sum_{j=1}^{M} \frac{\sigma_{j}}{\alpha_{j}}\right)}
$$

Using the definitions in (5), this expression leads to the solution for any system layout.

\section{A. Capacity and Admission Region}

Note that, assuming an unbounded optimum received power, the only valid solutions for (7) should accomplish that $S_{k}>0$ $\forall k$. Then, as both $\alpha_{k}$ and $\gamma^{\prime}$ are always positive real numbers, the following inequality must be accomplished:

$$
\sum_{k=1}^{M} \frac{\sigma_{k}}{\alpha_{k}}<1
$$

This inequality defines a $M$-dimensional region of the values of $\sigma_{k}$ that give valid received powers $S_{k}$ for all service classes. Then, this compact expression represents the so called admission region. Furthermore, the border of this admission region (expression (8) written as an equality) defines the maximum capacity of the system. However, note that $\sigma_{k}$ is a discrete variable, with steps of size $\nu_{k}$. Then, the border of the admission region actually is what is called a Pareto Front, and, for a general system layout, the values of $\sigma_{k}$ will not accomplish (8) with equality. Notice that for the case of a low number of services, this equation can be interpreted geometrically. Indeed, for $M=2$ it defines a straight line in the plane $\left(\sigma_{1}, \sigma_{2}\right)$, while for $M=3$ it defines a plane in a 3-dimensional space. In general, it defines a $M$-dimensional hyper-plane. Obviously, there are multiple combinations of the number of users per service that lead to valid solutions, and the boundary of the admission region is a discrete stair-shaped multi-dimensional border. 
TABLE I

PARAMETER VALUES FOR THE COMMON PARAMETERS

\begin{tabular}{|c|c|c|}
\hline Total available bandwidth & W & $5 \mathrm{MHz}$ \\
\hline Inter-cell ratio & $\beta$ & 0.4 \\
\hline Number of Repeaters & $N_{r}$ & 5 \\
\hline $\begin{array}{c}\text { Net Loss link } \\
\text { Base Station-Repeaters }\end{array}$ & $G_{R X, b} G_{T X, r i, b} L_{b, r i}$ & $-90 \mathrm{~dB}$ \\
\hline Base Station Noise Figure & $F_{b}$ & $5 \mathrm{~dB}$ \\
\hline Repeaters Noise Figure & $F_{r i}$ & $5 \mathrm{~dB}$ \\
\hline Repeaters Gain & $G_{r i}$ & $90 \mathrm{~dB}$ \\
\hline
\end{tabular}

TABLE II

Parameter values For the Different Service Classes

\begin{tabular}{|c|c|c|c|}
\hline & & Service 1 & Service 2 \\
\hline Quality & $\left(E_{b} / I_{t}\right)_{r e q}$ & $5.8 \mathrm{~dB}$ & $5.5 \mathrm{~dB}$ \\
\hline Bit Rate & $R_{b}$ & $64 \mathrm{kbps}$ & $128 \mathrm{kbps}$ \\
\hline Activity Factor & $\nu_{k}$ & 0.4 & 0.5 \\
\hline
\end{tabular}

\section{B. Practical Limitations}

In a real system, the values of $S_{k}$ are upper bounded. From expression (7) it is clear that the thermal noise addition provided by the repeaters in the system affects the system capacity as it increases the value of $\gamma^{\prime}$. Indeed, if we denote as $S_{\max }$ the maximum allowed received power, from (7) we can write that:

$$
\sum_{k=1}^{M} \frac{\sigma_{k}}{\alpha_{k}} \leq 1-\frac{\gamma^{\prime}}{\alpha_{k} S_{\max }}
$$

Then, comparing (9) with (8), we can explicitly see the capacity reduction of the system due to the bounded value of $S_{\max }$ and the contribution of the repeaters to the thermal noise level. This expression defines the new admission region. As a final observation on these expressions, notice that they can be applied with no restrictions on the characteristics of the system layout.

\section{EXAMPLE}

To enhance the understanding of the presented solution and to illustrate its behavior, this section shows the optimum values of $S_{k}$ for a two-service scenario, with parameter values taken from a representative real case. In this simple case, the admission region can be easily plot in a graph.

Let us suppose a system where the users belong to two possible service classes. In order to get example values, Table 1 shows the values for the parameters concerning the system layout, whereas Table 2 shows the settings for the two service classes. Solving (6) for every combinations of the number of users, Fig. 1 shows the needed received power for users belonging to service 1 . It is easy to verify that the boundary of the admission region corresponds to equation (8) with discrete variables $\sigma_{k}$. Its shape can be seen on the bottom of the graph. Note that the specific values of the needed receiver power depend on the parameter values. The presented results verify that they are in a range that fits with the state of the art technology for mobile communication devices.

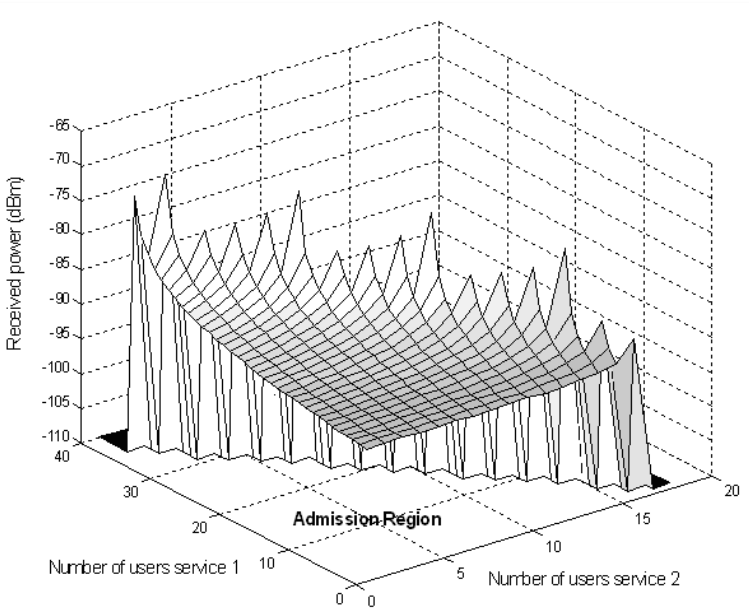

Fig. 1. Received power for users of service class 1 and admission region.

\section{CONCLUSION}

An analytical solution of the feasibility condition for the uplink of a CDMA mobile communication system for a general system deployment with the presence of repeaters in a multiservice scenario has been derived. A closed simple analytical expression allows to obtain the admission control region for any kind of users with heterogeneous QoS requirements. This solution shows explicitly the dependencies on the system settings of the capacity, in terms of number of users, focusing on the reduction provided by the increased noise level due to the presence of repeaters. Moreover, the solution can be applied to implement an admission control mechanism.

\section{REFERENCES}

[1] H. Holma and A. Toskala, Ed., WCDMA for UMTS Radio Access for Third Generation Mobile Communications, 2nd ed. New York: John Wiley \& Sons, 2002.

[2] J. Zander, "Performance of optimum transmitter power control in cellular radio systems," IEEE Trans. Veh. Technol., vol. 41, pp. 57-62, Feb. 1992.

[3] S. A. Jafar and A. Goldsmith, "Adaptive multirate CDMA for uplink throughput maximization," IEEE Trans. Wireless Commun., vol. 2, pp. 218-228, Mar. 2003.

[4] A. Baier, U. C. Fiebig, W. Granzow, P. Teder, and J. Thielecke, "Design study for a CDMA-based third-generation mobile radio system," IEEE $J$. Sel. Areas Commun., vol. 12, pp. 733-743, May 1994.

[5] W. Lee and D. Lee, "The impact of repeaters on CDMA system performance," in Proc. IEEE VTC 2000, pp. 1763-1767, 2000.

[6] M. Rahman and P. Ernstrom, "Repeaters for hotspot capacity in DSCDMA networks," IEEE Trans. Veh. Technol., vol. 53, pp. 626-633, May 2004.

[7] T. W. Ban, B. Y. Cho, W. Choi, and H.-S. Cho, "On the capacity of a DS-CDMA system with automatic on-off switching repeaters," in Proc. IEEE ICC01, pp. 780-784, 2001 